\title{
La contextualisation des recherches en environnement : une approche multiscalaire et multi-acteurs en Cévennes
}

\author{
Nathalie Couix ${ }^{1}$, Bernard Hubert ${ }^{2}$ \\ 1 Sciences des organisations, INRA, UMR1248 AGIR, 31326 Castanet-Tolosan cedex, France \\ 2 Écologue, INRA, Unité d'écodéveloppement, Site Agroparc, 84914 Avignon cedex 9, France
}

Les lecteurs de NSS sont familiers des travaux de Helga Nowotny (cf. NSS, 17, 1 [2009], 57-64) et, plus généralement, de ce processus de recherche que l'on qualifie de «mode 2 » (cf. NSS, 12, 1 [2004], 52-55). Dans cette autre façon de faire de la recherche, l'idée de contextualisation de la science est centrale, puisqu'elle renvoie aux transformations conjointes et successives du contexte comme des connaissances produites ainsi que du dispositif lui-même. Cet article en est l'illustration appuyée sur 15 ans d'observation participante, voire engagée. Il identifie les étapes qui mènent d'une recherche-intervention initiale à une situation où l'action et la production de connaissances entrent dans un dialogue mais aussi une confrontation rapprochés, qui obligent les différents acteurs de cette pièce à rebondissements à occuper le terrain de l'innovation.

La Rédaction

\section{Mots-clés :}

contextualisation de la recherche; recherche participative ; prévention des incendies de forêt ; transdisciplinarité ; Cévennes
Résumé - Depuis une trentaine d'années, plusieurs auteurs ont souligné une tendance de la recherche à être conduite dans ses contextes d'application. Ici, nous montrons à partir d'une étude de cas - le réaménagement du massif forestier du Rouvergue (Cévennes) -, que nous avons suivie durant treize ans, comment la recherche dans le domaine de la prévention des incendies de forêt et, plus généralement, de la gestion de l'espace peut être fortement ancrée dans ses contextes d'application. Nous nous intéressons conjointement à la transformation des différents contextes dans lesquels nous avons mis en place nos dispositifs de recherche successifs et à la transformation des orientations des recherches et des domaines de connaissances concernés. Cela nous conduit à approfondir la question des modalités concrètes du dialogue transdisciplinaire à instaurer entre recherche et société et celle d'une différenciation nécessaire des contextes de ce dialogue.

\section{Keywords:}

research

contextualisation;

participatory research; forest fire prevention; transdisciplinarity; Cévennes

\begin{abstract}
The contextualisation of research on the environment: a multi-scales and multi-actor approach in the Cévennes. Increasingly, the context in which research is conducted is being viewed as part of the research process itself as shown in the studies of many researchers over the past thirty years. Based on a case study in the French Cévennes in which we have been involved over some thirteen years, we illustrate how research on forest fire hazards prevention - and more generally land use management - could be contextualized at both the local and policy making levels. Central to the study was the design of a new landscape management project for a 4500 ha area recently ravaged by fire. The aim was to promote complementarities between forestry and livestock farming in order to create a landscape environment less sensitive to fire hazards. During the different project stages between 1986 and 2002 we participated in different working groups composed of locally involved stakeholders. These successive stages centered on different topics and working practices according to the group composition and the goals set by the participants. Our work highlighted the changes that occurred during this quite long period regarding the perception of
\end{abstract}

Auteur correspondant : N. Couix, Nathalie.Couix@toulouse.inra.fr 
issues, problems and questions identified by the stakeholders as well as their position regarding cooperation with researchers in a transdisciplinary process.

Our work focused on the interactions between the research dynamics and the changes that occurred in the different situations in which the institutional arrangements we introduced operated. In such situations where knowledge production and action are closely linked, cross-learning processes occur in all the participants including the researchers, causing relationships as well as the common topics and goals to evolve on a transdisciplinary base. More difficult is to identify how much the organizations involved could learn and capitalize from such processes involving their own agents even though this institutional level is mainly where policy making regarding such complex issues could become less sectoral and more systems-thinking and practicing in order to evolve adaptive capacities for change.

\section{Introduction}

Depuis une trentaine d'années, plusieurs auteurs ont proposé des modèles des processus de production de connaissances scientifiques et des modes de relation entre « recherche » et «société ». Ces modèles soulignent une tendance de la recherche à être conduite dans ses contextes d'application (Hessels et van Lente, 2008).

Dans cet article ${ }^{1}$, nous montrons, à partir d'une étude de cas, comment la recherche dans le domaine de la prévention des incendies de forêt et, plus généralement, de la gestion de l'espace peut être fortement ancrée dans ses contextes d'application et comment cela se traduit tant dans les actions concrètes menées sur le terrain et les politiques publiques correspondantes que dans les orientations des recherches et les domaines de connaissances concernés.

Le cas étudié est celui d'une opération d'aménagement à vocation de défense de la forêt contre les incendies $\left(\mathrm{DFCI}^{*}\right)^{2}$, en Cévennes, qui fait suite à un vaste incendie ayant dévasté 4500 hectares. Cette opération, démarrée en 1986, innovante à ses débuts, correspond à une transformation profonde des orientations en matière de DFCI - transformation à laquelle elle a contribué -, marquée par une participation beaucoup plus active des collectivités territoriales aux côtés de l'État et par le passage d'une vision en termes d'équipement pour la lutte active contre les incendies à une vision associant à la lutte la prévention des incendies. $\mathrm{Si}$, depuis, les politiques de DFCI se sont encore transformées, notamment en renforçant les actions en matière de gestion de l'espace, elles ont conservé cette orientation générale.

\footnotetext{
1 Ce travail a été conduit avec différentes formes de soutien financier selon les périodes considérées, en particulier celui du ministère de l'Agriculture et de la Forêt à son démarrage et celui du programme LEADER II « Espace Cévennes » et du Parc national des Cévennes pour la période 1999-2002.

2 Hormis DFCI, récurrent dans cet article, les sigles se rapportant spécifiquement à cette opération sont développés uniquement dans l'Encadré 1 . Ils sont suivis d'un astérisque la première fois qu'ils interviennent (dans le texte, puis dans le tableau). En revanche, les sigles plus génériques (tels que INRA ou GIS) sont simplement développés dans le texte.
}

Encadré 1. Sigles spécifiques rencontrés dans le texte et le tableau

CERPAM : Centre d'études et de réalisations pastorales Alpes-Méditerranée

CRPF : Centre régional de la propriété forestière

CTE : contrat territorial d'exploitation

DDAF : direction départementale de l'Agriculture et de la Forêt

DDE : direction départementale de l'Équipement

DFCI : défense de la forêt contre les incendies

DIREN : direction régionale de l'Environnement

GDA : groupement de développement agricole

IAM : Institut agronomique méditerranéen

ITOVIC : Institut technique de l'élevage ovin et caprin

OGAF : opération groupée d'aménagement forestier

ONF : Office national des forêts

PAC : Politique agricole commune

PAFI : plan d'aménagement forestier contre les incendies

PCPM : Périmètre cévenol du pin maritime

SDIS : service départemental d'Incendie et de Secours

SIME-LR : Service inter-chambres d'agriculture

Montagne Élevage - Languedoc-Roussillon

SIVOM : Syndicat intercommunal à vocations multiples

SIVU : Syndicat intercommunal à vocation unique

Nous avons suivi cette opération pendant treize années et y avons participéà différentes périodes. Ce suivi nous permet de bénéficier d'un recul suffisant pour apprécier la transformation des objets sur lesquels des connaissances ont été mobilisées ou produites par les agents des services techniques et administratifs impliqués dans l'opération et par la recherche, conjointement à une transformation de ses contextes.

Nous présentons dans une première partie le cadre d'interprétation sur lequel nous nous appuyons : il repose sur la notion de contextualisation de la recherche et de transdisciplinarité. Nous présentons ensuite l'étude 
$\mathrm{du}$ cas $\mathrm{du}$ réaménagement $\mathrm{du}$ massif forestier $\mathrm{du}$ Rouvergue (autour du col de Portes, dans les Cévennes gardoises) au travers de ce prisme. Cela nous conduit à approfondir la question des modalités concrètes du dialogue à instaurer entre recherche et société et celle d'une différenciation nécessaire des contextes de ce dialogue.

\section{Un cadre d'interprétation : contextualisation de la recherche et transdisciplinarité}

Nombreux sont les travaux sur les processus de production de connaissances scientifiques qui notent une interaction accrue entre «science » et « société » (Hessels et van Lente, 2008). Cette interaction se traduirait en particulier dans la participation de la société à la définition des agendas de la recherche. Si les courants de pensée analysés par les auteurs cités (New Production of Knowledge, Post-Normal Science, Triple Helix, etc. $)^{3}$ présentent des différences majeures dans leur interprétation des relations entre la recherche et la société, ils convergent à peu près autour de l'idée que la recherche est aujourd'hui souvent conduite dans ses contextes d'application ${ }^{4}$. Nowotny et al. (2001) parlent alors de « contextualisation » de la science. Pour eux, cette contextualisation est l'expression d'une forme de réflexivité au sens de Giddens (1984), par laquelle les discours deviennent constitutifs de la réalité qu'ils décrivent et contribuent ainsi à sa réorganisation. Pour ces auteurs, face à une incertitude grandissante au sein de la société, on assiste depuis un demi-siècle à un développement progressif d'un processus de communication entre la science et la société, c'est-à-dire à une articulation réflexive entre une " offre » et une "demande » de connaissances spécialisées. Les chercheurs ne sont plus les seuls à savoir quels problèmes doivent être résolus. Le « contexte », voire les multiples « contextes » répondent à la science, la sollicitent pour qu'elle apporte des solutions à des préoccupations d'individus, de groupes, d'organisations, et même de la société tout entière. La science cesse d'être

\footnotetext{
3 Hessels et van Lente (2008) proposent de mettre en perspective le modèle de Gibbons et al. (1994) dans son intégralité avec d'autres modèles des processus de production de connaissances scientifiques. Ici, seules les questions de la contextualisation et de la transdisciplinarité ( $c f$. infra) sont abordées.

4 Un des points de divergence est relatif au caractère plus ou moins récent de cette tendance. Pour certains, elle a toujours été. Pour d'autres, elle est plus récente. Shinn (2002), en particulier, dénonce dans le modèle de Gibbons et al. (1994) une rupture entre un mode traditionnel de production de connaissances où la science était isolée du reste de la société et le nouveau mode de production de connaissances, alors que le modèle de la «triple hélice » insisterait sur les continuités historiques des relations.
}

une activité pratiquée au sein de communautés closes et autocentrées dont seules les élites négocient avec l'extérieur, en particulier avec l'État ou l'industrie, les thèmes à aborder et les financements. Tout chercheur peut développer des relations de travail avec des partenaires socioéconomiques, ainsi que des collaborations scientifiques, y compris en dehors de sa communauté scientifique d'origine.

Pour Nowotny et al. (2001), il y a, selon les domaines, une contextualisation de la science plus ou moins forte en fonction de la force du signal émis par la société vers la science au sujet des contenus de la recherche. Réciproquement, cette contextualisation forte est rendue d'autant plus possible qu'il y a une forme de « consentement » des chercheurs à y répondre. Une contextualisation forte se traduit, entre autres, par une modification de la perception des chercheurs, et donc de leur définition des problèmes, de même que par des interactions continues qui, si elles durent suffisamment, peuvent se traduire par des approches nouvelles ou la mise en lumière de problèmes nouveaux. C'est d'ailleurs là un des enjeux portés par l'Agence nationale de la recherche (ANR) depuis sa création en 2005, non sans débats au sein de la communauté scientifique française.

Pour Shinn (2002), cette notion de contextualisation renvoie cependant à un rejet des différenciations institutionnelles entre la sphère de la recherche et del'Université et les autres sphères de la société. Dans un contexte de " communication inverse », ce serait alors la société qui déciderait du devenir du savoir et non plus les producteurs de ce savoir. Néanmoins, l'intérêt, selon nous, d'un prisme d'analyse tel que celui proposé par Nowotny et al. (2001) est de porter précisément l'attention sur la réciprocité des relations entre science et société - donc sur de véritables interactions -, au-delà des seules relations que les chercheurs établissent avec le reste de la société. De ce point de vue, leur modèle est aussi sensiblement différent des approches proposées par Latour (1995), où ce sont les chercheurs qui mobilisent différents «mondes » et non l'inverse. Ce faisant, ce prisme nous invite à dépasser l'analyse de nos dispositifs de recherche et des connaissances produites pour nous interroger sur les contextes dans lesquels ces dispositifs ont été élaborés et s'inscrivent. Il s'agit ainsi de rendre compte de l'importance de ces contextes dans la dynamique de la recherche et des'interroger sur les modalités du dialogue établi en leur sein.

Pour différents auteurs, l'établissement d'un dialogue effectif avec les acteurs de la société passe par la mise en place de démarches "problem-oriented" (Nowotny et al., 2001 ; Pohl et Hirsh Hadorn, 2008 ; Robinson, 2008), ce qui va souvent de pair avec des démarches interdisciplinaires, voire transdisciplinaires. Dans la littérature, en effet, les notions d'interdisciplinarité et de transdisciplinarité sont souvent reliées à la nature des problèmes qu'elles prétendent résoudre : ceux du monde « réel » 
(Hansson, 1999). Pour autant, toutes les recherches interdisciplinaires ne visent pas à répondre à des problèmes de la pratique. La distinction établie par Robinson (2008) entre «discipline-based interdisciplinarity » et «issue-driven interdisciplinarity » est intéressante. Alors que, dans le premier cas, l'interdisciplinarité est motivée par le souci de toujours repousser les limites des disciplines (un problème théorique à résoudre sur les fronts de connaissance, par exemple), dans le second, elle vise à résoudre des questions de société et des problèmes de la pratique. Les chercheurs concernés se trouvent alors à la frontière entre monde académique et monde " réel ». Ce type d'interdisciplinarité se caractérise en outre, selon l'auteur, par la participation active des acteurs extérieurs au monde de la recherche au processus de production de connaissances. Selon Robinson, cette notion $\mathrm{d}^{\prime} \ll$ issuedriven interdisciplinarity " se démarque de celle de transdisciplinarité par le fait que les chercheurs ne visent pas la production d'un nouveau cadre conceptuel. L'intégration des connaissances se veut d'abord guidée par le contexte concret de la recherche et par l'objectif de produire localement des connaissances utiles à l'action. Elle reste cependant très proche de la notion de transdisciplinarité telle que nous l'entendons ici, dans le sens où elle met en présence, dans une perspective de production de connaissances utiles à l'action, des acteurs (praticiens et chercheurs) confrontés à des limites disciplinaires différentes : thématiques pour les uns et plus académiques pour les autres. De même, cette notion est proche de celle développée par Nowotny, pour qui les connaissances produites transgressent les limites entre les disciplines, mais aussi celles du système traditionnel des sciences (Nowotny et Leroy, 2009).

Nous nous proposons dans la suite d'adopter ce prisme pour revisiter a posteriori l'opération de réaménagement du col de Portes. Le suivi que nous en avons fait nous a permis, en effet, d'en suivre les évolutions conjointement à celles des contextes dans lesquels elle a pris place, dont celui des politiques de prévention des feux de forêt et celui de la montée en puissance des questions environnementales.

\section{L'opération « col de Portes »: un cas de contextualisation forte}

\section{Au départ : une quasi-injonction de collaboration entre chercheurs et acteurs locaux}

Jusqu'aux lois de décentralisation (1982), l'État était le seul responsable de la protection de la forêt contre les incendies et de la prévention des risques «naturels ». Les principales orientations de sa politique en la matière étaient la sensibilisation du public, afin de limiter les départs de feux, et l'équipement des massifs, destiné à faciliter la lutte active contre les feux (pare-feux, pistes, citernes, etc.). D'importants incendies, survenus au début des années 1970, ayant mis en évidence la nécessité d'une continuité spatiale de ce type d'aménagement pour le rendre efficace, $l^{\prime}$ État mit en place de vastes périmètres de protection, d'un seul tenant, dans des sites très exposés au risque. Sept périmètres pilotes ont ainsi vu le jour, dont un dans le massif du Rouvergue ${ }^{5}$ en Cévennes.

En 1979, un vaste incendie détruisit une partie du périmètre pilote des Maures. En septembre 1985, 4500 hectares du périmètre cévenol de pins maritimes partirent en fumée en deux jours. La pertinence des actions conduites jusque-là, tant sur un plan national que localement, fut remise en cause. Au lendemain de l'incendie, en effet, les acteurs locaux, très marqués par ce qu'ils vivaient comme une catastrophe, se sont retournés, chacun avec ses propres propositions de solution, vers l'État pour qu'il assume ses responsabilités. Le ministre chargé de la Forêt s'adressa alors à l'Institut national de la recherche agronomique (INRA) ; il confia à Bernard Hubert, à l'époque responsable d'une nouvelle unité de recherches consacrée aux systèmes sylvopastoraux méditerranéens, la mission de présider et d'animer un groupe de travail réunissant chercheurs et techniciens locaux pour élaborer des propositions concrètes de réaménagement du site (Tab.). Le groupe devait aussi produire des propositions méthodologiques relatives à la prévention de ces sinistres, à transmettre au ministère après validation par un comité de pilotage, le « groupe élargi ». Ce faisant, le ministre manifestait sa volonté de voir le problème traité rapidement, $\mathrm{d}^{\prime}$ une manière innovante et reproductible. Il s'agissait en outre d'engager une réelle transformation de la politique de DFCI et donc de disposer d'autres connaissances que celles sur lesquelles les politiques en vigueur reposaient.

$C^{\prime}$ est donc dans un contexte de remise en cause profonde des actions conduites jusque-là pour prévenir les incendies de forêt qu' une collaboration entre chercheurs et acteurs locaux s'est mise en place dans ce secteur, sur une quasi-injonction du ministère et en réponse aux pressions des acteurs locaux : élus d'une région sensible (la zone minière d'Alès en cours de fermeture), agents de l'administration, personnalités politiques...

\section{D'intenses échanges au sein d'un dispositif distribué}

La première étape du travail a été la mise en place d'un dispositif distribué ${ }^{6}$ permettant à la fois la confrontation de différentes formes de connaissances - établies (knowledge) ou produites dans l'action (knowing) - pour faire émerger des solutions innovantes et la légitimation de ces dernières par les principales institutions (Tab.).

\footnotetext{
5 Massif presque monospécifique de pins maritimes, du fait de leur introduction, au siècle dernier, à des fins de production d'étais pour les galeries des mines d'Alès.

6 Constitué de groupes aux activités différentes.
} 


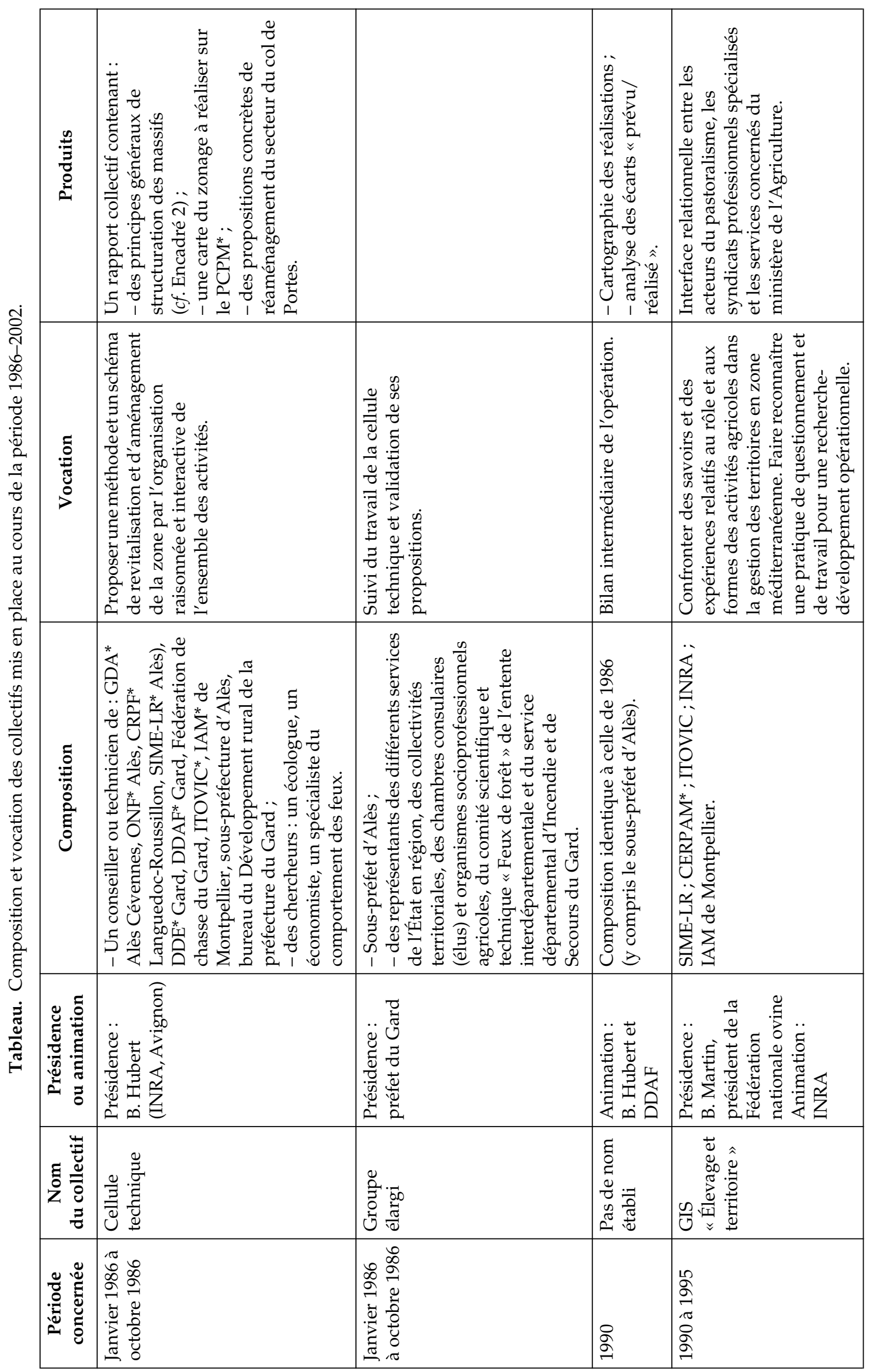




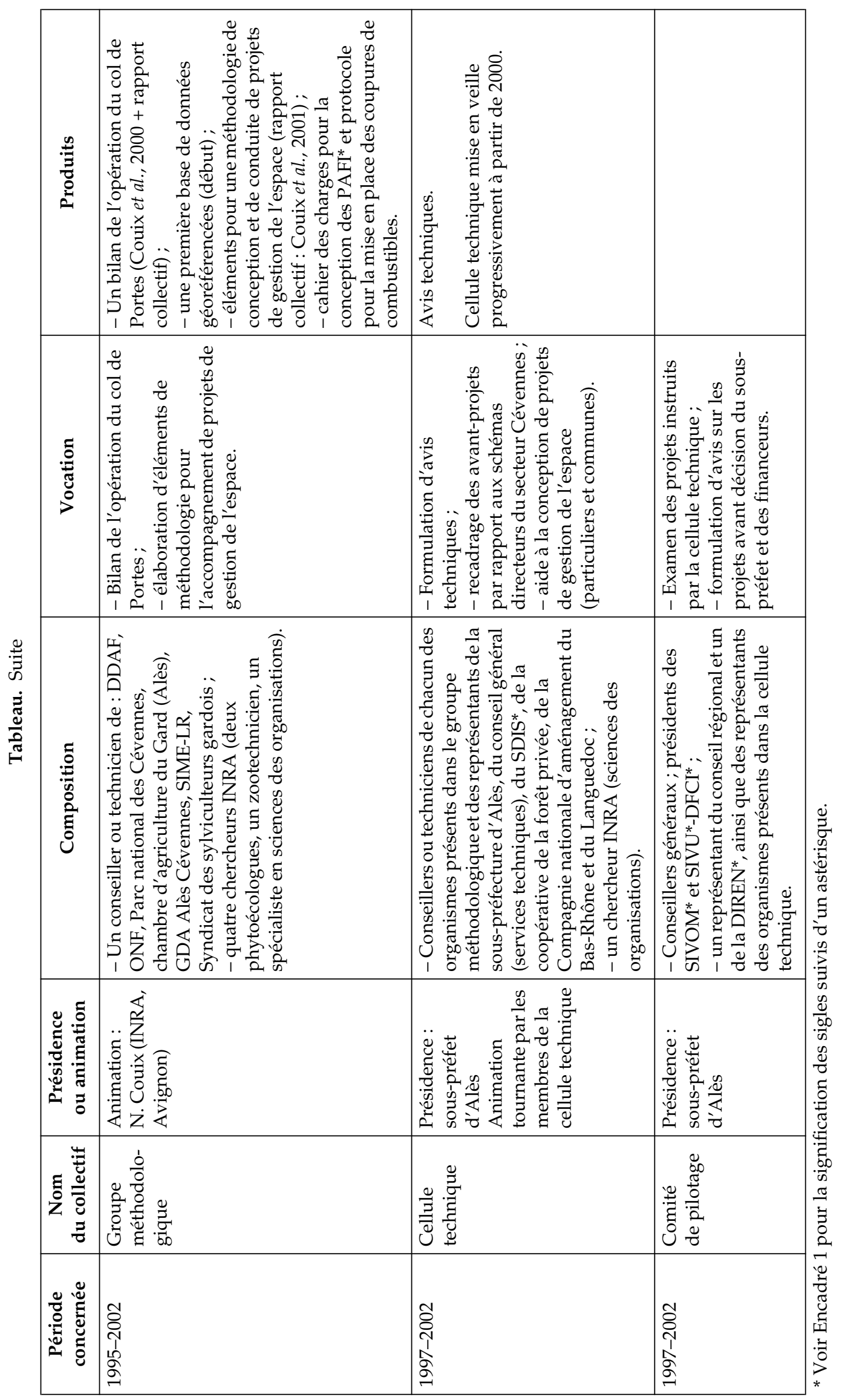


La commande du ministre était en effet que les différents partenaires techniques en charge de la DFCI travaillent ensemble, ce qui jusque-là n'aurait pas été imaginable, les relations entre forestiers, agriculteurs-éleveurs et chasseurs, ainsi qu'entre les services techniques correspondants étant très conflictuelles.

Dans ce contexte, les membres de la cellule technique ont reformulé le problème de la prévention des incendies, puis élaboré de nouveaux concepts d'action ${ }^{7}$. Le groupe s'est engagé dans l'analyse de la situation historique ayant permis le développement d'un tel incendie et dans la caractérisation des facteurs favorables à l'extension rapide des feux de forêt. Sur cette base, les réflexions se sont portées vers la recherche de solutions durables, fondées sur la mobilisation des ressources locales, pour créer un maillage pérenne du couvert végétal générant une hétérogénéité des masses de végétation défavorable à l'extension des incendies. Les travaux se sont alors orientés vers les fonctionnalités du territoire et, en corollaire, les formes de combinaison entre l'élevage et la foresterie susceptibles de produire un tel paysage sur le long terme. Des concepts d'action ont ainsi été produits (territoire en mosaïque, zonage des aménagements, etc.).

Après six mois de travail, les réflexions de la cellule technique ont abouti à des propositions d'aménagement présentées dans un rapport final. Soumis au groupe élargi et remis au préfet, ce rapport, rédigé à plusieurs mains, exprime l'accord des participants sur les propositions (Encadré 2). Celles-ci ont ensuite guidé les opérations (Collectif, 1986). Puis, lorsque la cellule technique a été dissoute, le préfet a demandé aux différents services de mettre en place cet aménagement en fonction de leurs compétences respectives.

Parallèlement, cette opération, considérée comme exemplaire de la transformation de la politique de DFCI, a fait l'objet d'une mise en scène politique pendant plusieurs années : visites de ministres au cours desquelles les chercheurs étaient mobilisés et colloques scientifiques. La " méthode Hubert »-c'est ainsi que techniciens et élus locaux appelaient les propositions de la cellule technique - a fait tache d'huile dans le département du Gard et la région.

L'opération «col de Portes », favorisant un dialogue assez intense entre recherche et " société ", a ainsi contribué à une transformation en profondeur des politiques

\footnotetext{
7 Au sens d'Hatchuel et Weil (1999), c'est-à-dire un ensemble de mots qui désignent un objet, un produit, etc., dont la mise en œuvre concrète nécessite la production de connaissances nouvelles. Au cours de projets de conception, partant de concepts d'action, des connaissances nouvelles vont être produites pour en permettre la mise en œuvre. Ces connaissances vont en retour induire une transformation des concepts d'action dans le sens d'une plus grande adaptation à celle-ci. C'est ainsi une dynamique des concepts d'action et des connaissances qui s'enclenche.
}

\section{Encadré 2. Principales propositions de la cellule} technique

Les principes énoncés visaient une restructuration fondée sur un zonage qui différencie trois types d'espace en fonction de leur caractère plus ou moins stratégique eu égard au développement et à la circulation des incendies. Ont ainsi été identifiées :

- des zones 1 : zones constituant les circuits privilégiés de départ et de propagation des incendies, où la gestion doit être très stricte, et zones d'arrêt ;

- des zones 2 : zones d'appui à la zone 1 ; elles doivent faciliter les interventions en zone 1 et diminuer les risques d'éclosion. Une gestion soignée par les activités sylvicoles et pastorales y est prévue ;

- des zones 3 : zones moins vulnérables, « libres» de toute contingence, si les deux premières zones ont bien été aménagées selon les recommandations émises.

L'ensemble s'appuyait sur une articulation des activités forestières et d'élevage concrétisée par des cahiers des charges réciproques et une répartition des moyens financiers bien plus intégrée que cela ne se pratiquait dans la tradition de l'aménagement rural français.

Ce modèle de structuration générale a été considéré comme innovant en ce qu'il contribuait à établir une nouvelle conception - formalisée et donc publiable ${ }^{\mathrm{a}}$ des aménagements préventifs des incendies de forêt : - fondée sur des mosaïques de zones boisées (de différentes essences et de différents âges) et d'espaces ouverts, inscrites dans le paysage en tenant compte des pentes et des systèmes de vents dominants (Delabraze et al., 1991 ; Hubert et al., 1993 ) ;

- reposant sur l'association d'activités économiques (la forêt et l'élevage) articulant au cours du temps leurs interventions sur ce territoire en mosaïque (Renaud et al., 1990 ; Guérin et al., 1991).

${ }^{a}$ Par des membres du groupe de travail ou des experts associés à ses réflexions.

de DFCI. Elle a impulsénotamment le passage d'une politique très centralisatrice de l'État à une politique associant les collectivités, territoriales et locales ${ }^{8}$, et l'ensemble des acteurs locaux.

\section{Une transformation progressive des questions de recherche}

Après la dissolution de la cellule technique, les relations entre recherche et société changent de nature. Un dialogue s'instaure et s'inscrit dans le temps (Tab.). Cela

\footnotetext{
8 La loi forestière de 1985 développait déjà le pouvoir des élus locaux en rendant les débroussaillements obligatoires dans les communes «à risque », sous la responsabilité des maires.
} 
se traduit par une transformation des questions et des produits de la recherche: d'interrogations en termes d'organisation des activités dans l'espace et dans le temps, le dialogue va s'ouvrir aussi à des questions d'action collective.

\section{Des contenus techniques à leur mise en œuvre}

Sur le terrain, la mise en œuvre des propositions du schéma d'aménagement se révèle difficile dès les premières années. Le passage d'une politique de sensibilisation et d'équipement à une problématique concertée d'aménagement n'est pas si simple... Aussi, en 1990, alors que la tendance était au développement des procédures d'évaluation des politiques publiques et que la politique de prévention des incendies cherchait à affiner ses principes (mission "Cochelin » du Conseil général du génie rural, des eaux et des forêts), une tentative d'autoévaluation du projet en cours a été lancée, par B. Hubert et un représentant de la $\mathrm{DDAF}^{*}$, pour identifier et résoudre les difficultés rencontrées, voire réajuster les objectifs de celui-ci (Tab.). Cependant, cette tentative de bilan intermédiaire n'a pas permis le travail réflexif attendu et ce, pour diverses raisons : relations trop peu stabilisées entre les techniciens locaux et les agents des services administratifs pour permettre une réflexion sur les pratiques de chacun et les modes de fonctionnement des différentes institutions ; manque de disponibilité des chercheurs.

Ce bilan a néanmoins permis aux parties prenantes de constater que de nombreuses actions avaient été engagées, mais qu'il y avait eu des difficultés inhérentes à l'articulation des activités. En particulier, la comparaison des cartes "prévu/réalisé » a révélé des discontinuités dans les aménagements. Celles-ci traduisaient les modes d'intervention des techniciens pour élaborer les actions concrètes avec les agriculteurs et les propriétaires forestiers. Les propositions de la cellule technique de 1986 prévoyaient de mettre en place le schéma en intervenant par petites entités géographiques au sein desquelles toutes les activités auraient été prises en compte. Mais cela ne correspondait pas à la logique d'intervention des techniciens. Le schéma, qui avait joué un rôle de concept intermédiaire (Teulier et Hubert, 2008) au sein de la cellule technique, était devenu une norme à respecter, mobilisée par les uns et les autres pour réfléchir individuellement sur les actions sylvicoles ou agricoles. Aussi, ce bilan a révélé à tous la nécessité de produire des connaissances sur les procédures d'action collective afin de mettre en œuvre les concepts d'action élaborés en 1986.

\section{Un thème en émergence : la notion de concertation}

La volonté d'adopter une démarche concertée, pour les institutions et les techniciens du monde agricole au moins, n'est plus spécifique à cette opération. À cette période, se multiplient les mesures prises pour favoriser un dialogue entre les différentes parties prenantes $\mathrm{du}$ monde agricole et de l'environnement: la notion de concertation devient un maitre mot tant au sein de la " société » que dans le monde de la recherche, comme en témoignent un nombre croissant de publications et certains appels à projets (en particulier du ministère en charge de l'Environnement). Pris dans cette mouvance, les différents agents du secteur des Cévennes alésiennes sont confrontés, au cours des années 1990, à une diversité de mesures et d'outils (OGAF* Environnement, CTE*, Natura 2000, etc.), ainsi qu'à une diversité de structures de concertation (commission Fonds de gestion de l'espace rural, comité Natura 2000, comités de pilotage, etc.).

C'est à cette époque qu'à la suite de nouvelles rencontres avec certains acteurs locaux, N. Couix a proposé aux techniciens locaux de reformer un groupe de travail afin de tirer des enseignements plus généraux de l'opération «col de Portes » (Tab.). Dès les premières réunions, la plupart des techniciens ont exprimé des besoins de coordination entre les interventions des différents partenaires de la gestion de l'espace, notamment :

- une coordination des projets entre eux à l'échelle de l'ensemble du secteur des Cévennes alésiennes ;

- une coordination des techniciens au sein d'un même projet afin de favoriser des actions concertées mêlant les différentes activités.

Au-delà, ils ont souhaité traiter de leurs modes d'organisation, en tant qu'agents et entre institutions, pour parvenir à une gestion concertée de l'espace. Cela changeait donc considérablement le contenu des échanges entre chercheurs et praticiens.

La première proposition des techniciens locaux fut alors de remettre en place une structure réunissant les institutions impliquées dans l'application des politiques de DFCI. Cette nouvelle cellule technique, accompagnée d'un comité de pilotage, a été constituée en 1997 (Tab.). Après trois ans de fonctionnement, elle s'est révélée peu intéressante du fait même de la multiplicité des structures de concertation existantes à cette période. Le relatif échec de cette structure a alors permis de réfléchir collectivement à une nouvelle conception de l'accompagnement de ces projets. L'analyse de la démarche mise en œuvre dans le cadre de l'opération " col de Portes », ainsi que d'autres projets en cours à cette période, a montré que les échanges entre les différents techniciens, au sein des nombreuses structures d'interaction auxquelles ils participaient, portaient surtout sur le contenu technique des projets (nature des actions à conduire et modalités techniques de mise en œuvre de ces actions). Très peu d'échanges étaient consacrés aux modalités de coordination nécessaires pour accompagner ces projets. Cet accompagnement restait l'affaire de chacun dans son domaine spécifique. La question a alors été celle d'un accompagnement global du projet, et les techniciens se sont interrogés avec les chercheurs sur 
la manière dont ils allaient, en tant que " collectif », aider les porteurs de projets à concevoir leurs actions et les accompagner dans leur démarche. Les pratiques d'intervention des différents techniciens ont alors été mises en discussion dans le cadre des projets concrets, afin de les rendre plus cohérentes entre elles. Les modalités pour « agir ensemble » se sont ainsi trouvées peu à peu au cœur des échanges entre chercheurs et praticiens (Couix, 2002). $C^{\prime}$ est bien à une transformation en profondeur des questions de recherche que ces interactions sont parvenues.

Grâce aux expériences acquises et aux réflexions conduites - notamment avec la recherche -, les différents services techniques et administratifs ont ensuite engagé un travail de formalisation de méthodologies de conception et de conduite de ces plans, précisant aussi les modes d'intervention et les rôles de chacun : protocole pour les coupures de combustible, cahier des charges pour la conception des $\mathrm{PAFI}^{*}$, par exemple.

\section{Une tentative d'institutionnalisation à travers la constitution d'un groupement d'intérêt scientifique}

De façon à s'extraire un peu de la situation locale cévenole, comme d'autres en Languedoc ou en Provence dans lesquelles plusieurs partenaires étaient engagés, nous avons contribué à la constitution d'un groupement d'intérêt scientifique (GIS), dénommé « Élevage et territoire » (Tab.). Celui-ci a été créé en 1990 à l'initiative des principales institutions impliquées dans des opérations de terrain similaires des deux côtés du Rhône, avec les services du ministère concernés et les représentants de la profession agricole. L'objectif était de constituer un lieu de capitalisation des expériences concrètes, afin d'agir à un autre niveau de représentation que les parties prenantes locales. Participaient ainsi aux activités du GIS des représentants des directions concernées du ministère, de la direction scientifique de l'INRA et des organisations professionnelles agricoles nationales. Il s'agissait de montrer qu'il y avait dans ces questions de réintroduction de l'élevage dans les espaces boisés méditerranéens des enjeux cognitifs, politiques et stratégiques, tout en étant opérationnels en situation. C'est bien là que les concepts produits en situation peuvent être confrontés les uns aux autres et stabilisés, afin d'aider à la priorisation des différentes formes de connaissance (technique, organisationnelle, juridique, économique, etc.) nécessaires à leur mise en œuvre dans une diversité de cas. Ce GIS n'a pas produit ce qui en était attendu, mais il a permis l'émergence de nombreuses initiatives et projets entre les organismes de recherche-développement, la recherche et les services concernés des régions et du ministère en charge de l'agriculture.

Localement, on peut ainsi parler d'une articulation réflexive entre la recherche et une partie de la société : le long dialogue entre ces deux sphères, à l'initiative de l'une ou de l'autre selon les périodes, s'est bel et bien traduit par des changements en profondeur des contenus de la recherche et de certaines pratiques des techniciens et agents de développement. Au plan national, cette opération au départ très innovante s'est inscrite dans une tendance plus générale. Et si cette tendance a conduit, aux côtés des politiques publiques, à la production d'un grand nombre de guides méthodologiques pour la concertation, ceux-ci sont restés longtemps discrets quant aux modalités d'accompagnement des projets et surtout aux procédures qui peuvent permettre aux différents partenaires « d'agir ensemble».

\section{Faire avec une diversité d'acteurs et de contextes}

Le cas de l'opération du col de Portes illustre bien l'idée que la recherche est de plus en plus conduite dans son contexte d'application et que des formes de dialogue se sont instaurées entre la science, les politiques, l'industrie, la société civile, etc. Au-delà, le cas est intéressant du point de vue des modalités du dialogue à l'origine de cette contextualisation, modalités sur lesquelles les auteurs consultés passent rapidement. Comment un processus de communication, c'est-à-dire de « mise en commun » et de reconstruction du sens (Watzlavick et al., 1979 ; Ison, 2010) peut-il réellement s'instaurer entre science et société ?

\section{Des approches transdisciplinaires au sein de dispositifs de type "recherche-intervention "}

Au cours de ces treize années, nos relations avec les acteurs de terrain en Cévennes ont connu des formes diverses. Néanmoins, les dispositifs au sein desquels nous avons travaillé avec les techniciens nous ont toujours permis de prendre une part active à la construction même de l'action. Une hypothèse à la base de nos démarches était que nous ne pouvions pas élaborer de connaissances pertinentes pour la gestion de l'espace sans être parties prenantes, au moins pour un temps, des actions conduites localement. Un corollaire étant pour nous que, pour produire des connaissances pertinentes, les dispositifs de recherche mis en place se devaient d'être participatifs, voire transdisciplinaires, comme nous les qualifierions aujourd'hui (cf. supra). D'une manière générale, les dispositifs élaborés ont visé à mettre des chercheurs de disciplines différentes et les divers praticiens dans une situation de knowing au sens de Cook et Brown (1999), c'est-à-dire de production de connaissances dans l'action en train de se faire, afin de résoudre des problèmes de la pratique, et pas seulement de mobiliser les connaissances déjà acquises (knowledge), ici ou ailleurs, sur le modèle d'une démarche de simple expertise. Et c'est la résolution de ces problèmes de la pratique 
qui a en partie guidé, au fil des travaux, la mobilisation plus ou moins importante des compétences tant scientifiques que professionnelles.

À l'origine, cette posture de recherche relevait plus $\mathrm{d}^{\prime}$ une position de principe que de la mise en application de théories formelles sur la production de connaissances. Les principes de la recherche-intervention proposés plus tard par Hatchuel (2000) caractérisent bien la démarche que nous avons tentée (au moins !) d'avoir au cours de cette recherche. Dans le cas présentéici, cette posture nous a permis de suivre et d'accompagner la transformation des questionnements de nos partenaires, transformant ainsi nos propres questions, dans un processus d'apprentissage réciproque. C'est en ce sens que nous parlons de contextualisation forte de la recherche, en insistant d'ailleurs davantage que ne le font Nowotny et al. (2001) sur la réciprocité des transformations : non seulement la recherche transforme ses questions en fonction des contextes, mais, au niveau local comme global, il y a aussi transformation des discours et des pratiques.

De même, quelle que soit la période, les chercheurs pas plus que les techniciens n'avaient une idée précise de ce vers quoi ils allaient ni de ce sur quoi il fallait produire de la connaissance. C'est cette posture inhabituelle, voire très inconfortable pour un chercheur, qui a rendu possible le dialogue. Les chercheurs se sont plus trouvés en situation d'animer une réflexion et de favoriser une confrontation des connaissances, que de produire des connaissances scientifiques au sens classique du terme. Et, ce faisant, ils ont accompagné une transformation des modes d'action des différents partenaires et une coproduction de connaissances nouvelles pour chacune des parties.

\section{Une démarche exploratoire pour suspendre les discontinuités au sein d'un collectif d'acteurs hétérogènes...}

En 1986, devant l'hétérogénéité du groupe, relative aux positions sociales, aux modes de légitimité, aux connaissances, aux intérêts, B. Hubert a animé le travail de la cellule technique de manière à rendre les interactions un peu plus symétriques, c'est-à-dire à « suspendre » les discontinuités (cognitives, culturelles, etc.) au sein du groupe. Cela a été possible grâce à sa légitimité, conférée par le ministre, et à une certaine autorité (encore à l'époque !) du chercheur. Pour cela, il a fait le choix méthodologique d'une dynamique d'exploration collective et de recherche des concepts les plus opératoires pour changer la situation. La cellule technique a ainsi fonctionné comme un collectif exploratoire formellement engagé et légitimé dans la recherche de solutions innovantes à une question relativement partagée : Comment faire pour qu'un tel incendie ne se reproduise pas ? C'est ainsi que nous avons privilégié les confrontations sur le terrain et les discussions autour de nouveaux instruments comme des cartes, des bilans économiques d'exploitations, des posters sur les typologies des essences forestières, etc., de façon à obtenir, assez vite, une reformulation partagée de la question à traiter. En tant que chercheurs, nous avons préféré utiliser nos connaissances du domaine pour identifier et évaluer l'originalité des idées émises au cours des échanges, pour révéler les savoirs des autres afin de les rendre partageables ou inciter à la constitution de nouveaux savoirs communs, phase indispensable dans une démarche transdisciplinaire selon Pohl et Hirsch-Hadorn (2008), plutôt que d'orienter le choix de solutions. De même, au sein du groupe, nous avons peu à peu identifié des domaines de connaissance que nous avions besoin $\mathrm{d}^{\prime}$ acquérir pour traiter la question. Nous sommes alors convenus d'inviter des experts aux visites de terrain: pompiers, sécurité civile, exploitants forestiers, élus locaux, chercheurs spécialisés (forêt, incendies, pastoralisme, agroéconomie...), etc., afin de permettre une synchronisation cognitive (Darse et Falzon, 1996) des membres de la cellule technique sur les différentes facettes de la question à traiter.

\section{... pour lequel connaissances et actions sont indissolublement liées}

Plus tard, en 1995 et durant les six ans au cours desquels nous avons collaboré avec les techniciens locaux en Cévennes, le dispositif mis en place, les méthodologies mobilisées ainsi que le rôle des chercheurs se sont transformés en fonction des attentes de nos partenaires et de notre propre compréhension des processus étudiés. Cette transformation peut s'interpréter comme un passage progressif d'un dispositif visant une réflexion sur les pratiques effectives de mise en œuvre du schéma d'aménagement et ce qu'elles ont produit concrètement à un dispositif reposant à la fois sur des discussions « in practice » et « on practice ». Àl'usage, cette transformation s'est révélée féconde, en particulier du point de vue des sciences des organisations ${ }^{9}$. Plusieurs auteurs du courant des Practices Based Studies (comme Gherardi [2006] ou Cook et Brown [1999]) défendent l'idée du caractère intrinsèquement lié des processus de production de connaissances et des pratiques quotidiennes : pour eux, connaissance et action ne sont pas séparables. C'est bien ce que notre travail avec les techniciens locaux a aussi permis de révéler. Dans les contenus, les échanges sont passés de « ce qu'on a fait », « ce que cela a donné », « ce qu'on devrait ou pourrait faire » selon la situation à des échanges visant, dans des cas concrets de projets, des ajustements réciproques. Cela a permis à chacun, y

\footnotetext{
9 À cette époque, les questions ont plus concerné ce domaine scientifique que ceux des disciplines biotechniques. Le dispositif est resté malgré tout transdisciplinaire au sens retenu ici.
} 
compris aux chercheurs, de mieux comprendre ce que faisaient les autres « dans l'action » et de s'ajuster mutuellement, en fonction de la situation. Ces échanges et la nécessité d'agir ensuite ont permis d'identifier de nombreux sujets sur lesquels une discussion entre praticiens se révélait nécessaire en vue d'une collaboration ou sur lesquels des connaissances étaient à produire. L'objet de ces discussions n'était plus de parvenir à une vision partagée de ce que devaient être des modalités de mise en œuvre d'un schéma directeur, celui du col de Portes ou un autre. Il s'agissait de trouver, au cas par cas, comment s'ajuster. Ce faisant, cela a permis de mieux comprendre les convergences et les divergences entre les pratiques de chacun et d'identifier des modalités de coordination entre les activités des uns et des autres.

\section{Identifier des contextes pertinents pour construire différents types de dialogue}

L'analyse du cas du col de Portes nous conduit à souligner l'importance de distinguer les différents contextes au sein desquels un dialogue science-société a pu et peut se mettre en place. Ici, il est important de distinguer le contexte local correspondant aux acteurs du terrain, impliqués concrètement dans cette opération, à leurs histoires, à leurs réseaux, à l'évolution de l'environnement biophysique - comme la reprise de la végétation à la suite de l'incendie et la transformation des milieux sous l'effet des reboisements et des pratiques de pâturage, etc. Par ailleurs, nous distinguons un contexte plus général des politiques, des institutions, des représentants de l'État ou encore des législateurs, eux-mêmes pris dans la dynamique générale de l'émergence des problèmes d'environnement, des remises en cause et des réformes successives des politiques agricoles, permettant l'instauration de nouveaux instruments et outils de politiques publiques (Natura 2000, mesures agrienvironnementales de la $\mathrm{PAC}^{*}$, etc.). Si ces deux types de contexte interagissent entre eux ainsi qu'avec la recherche, comme nous l'avons $\mathrm{vu}$, ils ne connaissent pas pour autant les mêmes dynamiques et notamment pas les mêmes rythmes. De même, la communication ne se fait pas si aisément entre eux.

$C^{\prime}$ est la confrontation et l'ajustement réciproque entre ces deux contextes que visaient le GIS «Élevage et territoire » en tant qu'instance permanente d'échanges et d'initiatives, ainsi que les visites de terrain réalisées à l'occasion de colloques divers (Msika et Hubert, 1988) ou encore les visites de plusieurs ministres. Il s'agissait, d'une part, de montrer aux décideurs politiques la mise en œuvre, sur le terrain, des procédures qu'ils formalisaient à des échelles nationales (voire communautaires). Il s'agissait, d'autre part, de rappeler aux acteurs de terrain qu'au-delà de la réalité concrète de leur situation, ils étaient parties prenantes de dynamiques intellectuelles et politiques qui étaient celles d'un contexte général. Celui-ci pouvait leur paraître éloigné, mais il cadrait néanmoins les instruments réglementaires et juridiques qu'ils mobilisaient, ainsi que les fonds auxquels ils recourraient pour réaliser leurs projets. En trente ans, la déconcentration des pouvoirs politiques s'est souvent accompagnée d'un retrait partiel de l'État, renvoyant aux acteurs de proximité la gestion de la complexité de situations que des normes universelles n'étaient pas à même d'assurer. Cette dynamique s'est accompagnée d'un discours général - ici comme ailleurs - d'incitation à la participation des acteurs concernés, sans gérer la transition avec les politiques d'autorité précédentes ni proposer de cadre méthodologique approprié à une diversité de situations. Cela a favorisé le développement de structures intermédiaires (organisations locales, ONG de dimensions variables...), se posant en institutions médiatrices, plus à l'aise avec le discours participatif que ne le sont des collectifs hétérogènes constitués d'acteurs aux intérêts divergents.

En conclusion, nous soulignerons la nécessité de la réciprocité de l'apprentissage entre chercheurs et nonchercheurs pour permettre cette contextualisation de la science. S'il est devenu plus habituel pour nous, chercheurs, de nous interroger sur nos pratiques de recherche et sur la pertinence des connaissances produites, il est nécessaire aussi que les partenaires potentiels de cette recherche jouent le jeu pour que le dialogue s'établisse réellement et soit productif (Couix et Hubert, 2000 ; Gonzalo-Turpin et al., 2008). Suspendre les discontinuités entre agents et entre niveaux institutionnels ne va pas de soi. En Cévennes, on fait bien la différence à ce sujet entre les agricoles et les forestiers, ces derniers, à l'époque, n'ayant pas vraiment remis en cause leur expertise et leur position. Nous avons également tenté d'articuler ces différents niveaux de contextualisation, préoccupés chacun par des priorités, des enjeux, des environnements (naturels, politiques, économiques, sociaux, culturels...) différents. Mais, si on a pu mettre en évidence quelques effets des apprentissages au niveau des acteurs locaux mis en interaction (chercheurs compris !), il est plus difficile d'aborder la question de l'apprentissage au sein des organisations et des institutions elles-mêmes, des ministères et de leurs services ou des organisations professionnelles agricoles, par exemple. Comment s'y capitalisent les acquis de leurs agents - souvent, en outre, de passage ? Or, c'est aussi là que s'acquièrent les caractères génériques de durabilité et de transportabilité des propositions innovantes. C'est là une question pour la recherche sur l'action collective qui reste, de notre point de vue, encore ouverte, et c'est un rôle que doivent pouvoir jouer les chercheurs, en tant que passeurs entre les mondes locaux du terrain et ceux des sphères décisionnelles de l'action publique. 


\section{Références}

Collectif, 1986. Reconstitution en Cévennes d'un massif forestier vivant : intégrer les activités pour un espace diversifié. Rapport de la cellule technique pour le réaménagement du périmètre incendié des Cévennes, sous-préfecture d'Alès.

Cook, S.D.N., Brown, J.S., 1999. Bridging epistemologies: The generative dance between organizational knowledge and organizational knowing, Organization Science, 10, 4, 381400.

Couix, N., 2002. Concerted approach to land-use management: Developing common working procedures. A Cévennes case study (France), Land Use Policy, 19, 75-90.

Couix, N., Hubert, B., 2000. Promoting collective learning in a land use management project. Thirteen years' experience in researcher-technician partnership in the Cévennes (France), in LEARN Group [Cerf, M., Gibbon, D., Hubert, B., Ison, R., Jiggins, J., Paine, M., Proost, J., Roling, N.] (Eds), Cow Up a Tree. Knowing and Learning for Change in Agriculture: Case Studies from Industrialised Countries, Paris, INRA Éditions, 121-140.

Couix, N., Étienne, M., Léouffre, M.C., Napoleone, M., Marjollet, G., Brosse, E., Schuller, F., Bolchert, C., Detry Fouque, P., Lécuyer, D., 2000. L'opération pilote du col de Portes (Gard). Quels enseignements treize ans après ?, Forêt méditerranéenne, XXI, 3, 308-312.

Couix, N., Étienne, M., Napoleone, M., 2001. Gestion concertée en Cévennes alésiennes. Rapport final de l'action L2 0202 10012 LEADER II «Espace Cévennes », Montpellier, INRA, UMR Innovation / Avignon, INRA, Unité d'écodéveloppement.

Darses, F., Falzon, P., 1996. La conception collective: une approche de l'ergonomie cognitive, in Terssac, G. de, Friedberg, E. (Eds), Coopération et conception, Toulouse, Octares, 123-135.

Delabraze, P., Hubert, B., Oswald, H., Valette, J.-C., 1991. L'aménagement de la forêt pour une meilleure prévention, La Recherche, 234, 902-907.

Gherardi, S., 2006. Organizational Knowledge: The Texture of Workplace Learning, Malden (MA), Blackwell.

Gibbons, M., Limoges, C., Nowotny, H., Schwartzman, S., Scott, P., Trow. M., 1994. The New Production of Knowledge: The Dynamics of Science and Research in Contemporary Societies, London, SAGE Publications.

Giddens, A., 1984. The Constitution of Society, Cambridge (UK), Polity Press.

Gonzalo-Turpin, H., Couix, N., Hazard, L., 2008. Rethinking partnership with the aim of producing knowledge with practical relevance: A case study in the field of ecological restoration, Ecology and Society, 13, 2, 53.

Guérin, G., Hubert, B., Strohl, J., 1991. Articuler activités agricoles et forestières dans l'arrière-pays méditerranéen. Réflexion effectuée à partir du travail au col de Portes en Cévennes, Bulletin technique d'information, 4, 49-58.

Hansson, B., 1999. Interdisciplinarity: For what purpose?, Policy Sciences, 32, 339-343.
Hatchuel, A., 2000. Intervention research and the production of knowledge, in LEARN Group [Cerf, M., Gibbon, D., Hubert, B., Ison, R., Jiggins, J., Paine, M., Proost, J., Roling, N.] (Eds), Cow Up a Tree. Knowing and Learning for Change in Agriculture: Case Studies from Industrialised Countries, Paris, INRA Éditions, 55-68.

Hatchuel, A., Weil, B., 1999. Design-oriented organizations. Towards a unified theory of design activities, in Gardiner, G., Gregory, M., Karlsson, C. (Eds), 6th International Product Development, Management conference, Cambridge (UK), July 56, 1999, Brussels, EIASM.

Hessels, L.K., van Lente, H., 2008. Re-thinking new knowledge production: A literature review and a research agenda, Research Policy, 37, 4, 740-760.

Hubert, B., Rigolot, É., Turlan, T., Couix, N., 1993. Forest fire prevention in the Mediterranean Region. New approaches to agriculture-environment relations, in Brossier, J., de Bonneval, L., Landais, É., (Eds), System Studies in Agriculture and Rural Development, Paris, INRA Éditions.

Ison, R., 2010. Systems Practice: How to Act in a Climate-Change World, London and New York, Springer / Milton Keynes (UK), Open University.

Latour, B., 1995. Le Métier de chercheur: regard d'un anthropologue, Paris, INRA Éditions.

Msika, B., Hubert, B. (Eds), 1988. Agriculture et forêt en région méditerranéenne française: actes du colloque organisé par l'INRA et le ministère de l'Agriculture et de la Forêt, direction de l'Espace rural et de la Forêt, Toulon (France), octobre 1987, [Paris], INRA Éditions.

Nowotny, H., Scott, P., Gibbons, M. 2001. Re-Thinking Science: Knowledge and the Public in an Age of Uncertainty, Cambridge (UK), Polity Press.

Nowotny, H., Leroy, P., 2009. Helga Nowotny: An itinerary between sociology of knowledge and public debate. Interview by Pieter Leroy, Natures Sciences Sociétés, 17, 1, 57-64.

Pohl, C., Hirsch-Hadorn, G., 2008. Methodological challenges of transdisciplinary research, Natures Sciences Sociétés, 16, 2, 111-121.

Renaud, J.-P., Godzinsky, F., Maurel, M., 1990. Le réaménagement du massif du Rouvergue dans les Cévennes gardoises après le grand incendie de septembre 1985, Revue forestière française, numéro spécial « Espaces forestiers et incendies », 307-317.

Robinson, J., 2008. Being undisciplined: Transgressions and intersections in academia and beyond, Futures, 40, 70-86.

Shinn, T., 2002. Nouvelle production du savoir et triple hélice : tendances du prêt-à-penser les sciences, Actes de la recherche en sciences sociales, 141, 21-30.

Teulier, R., Hubert, B., 2008. Des concepts intermédiaires pour la conception collective. Les situations d'action collective avec acteurs hétérogènes, in Mélard, F. (Ed.), Écologisation : objets et concepts intermédiaires, Bruxelles, Peter Lang, 163186.

Watzlawick, P., Beavin, J., Jackson, D., 1979. Une logique de la communication, Paris, Le Seuil.

Reçu le 17 septembre 2009. Accepté le 6 octobre 2011. 